\title{
Observance of Improved Magneto-resistance and Magnetic Entropy Change in $\mathrm{La}_{0.7}\left(\mathrm{Ca}_{0.2} \mathrm{Sr}_{0.1}\right) \mathrm{MnO}_{3}$ :Pd Composite
}

\author{
Ramesh Chandra Bhatt • P.C. Srivastava • \\ S.K. Agarwal • V.P.S. Awana
}

Received: 25 November 2013 / Accepted: 28 November 2013 / Published online: 15 December 2013

(C) Springer Science+Business Media New York 2013

\begin{abstract}
Magneto-resistive and magneto-caloric properties of polycrystalline $\mathrm{La}_{0.7}\left(\mathrm{Ca}_{0.2} \mathrm{Sr}_{0.1}\right) \mathrm{MnO}_{3}$ (LCSMO) and $\mathrm{La}_{0.7}\left(\mathrm{Ca}_{0.2} \mathrm{Sr}_{0.1}\right) \mathrm{MnO}_{3}: 10 \% \mathrm{PdO}$ (LCSMO:Pd10) composites sintered at $1400{ }^{\circ} \mathrm{C}$ have been investigated. Rietveld refinement of their X-ray diffraction $(X R D)$ patterns confirms the single-phase crystalline structure with orthorhombic Pbnm space group, showing no significant change in their lattice parameters with Pd addition. Disappearance of the low temperature resistivity hump in the Pd composite has been attributed to the suppression of the grain boundary effect and the conducting channels due to the presence of metallic Pd. While the Curie temperature $T_{c}$ remained nearly unchanged, the peak value of the temperature coefficient of resistance (TCR) increased in the composite. $T C R_{\text {MAX }}$ increased to $6.4 \%$ (at $308 \mathrm{~K}$ ) in the composite from $2 \%$ (at $305 \mathrm{~K}$ ) for the pristine LCSMO. Magnetoresistance $(M R)$ and magnetic entropy change $\left(\Delta S_{M}\right)$ also increased markedly in the composite material. This could be ascribed to the observed sharpness of both the magnetic and resistive transitions resulting from better grain connectivity. Maximum $M R$ of $12.9 \%(1 \mathrm{~T})$ and $19.6 \%(2 \mathrm{~T})$ has been observed close to its $T_{c}(309 \mathrm{~K})$ in the pristine LCSMO. These values increased to $24.1 \%(1 \mathrm{~T})$ and $33.9 \%(2 \mathrm{~T})$ with the addition of PdO. The maximum values of $\left|\Delta S_{M}\right|$ are found to be $4.4,8.2$ and $11.7 \mathrm{~J} \mathrm{~kg}^{-1} \mathrm{~K}^{-1}$ at field values of 1, 2 and 3 T, respectively, for LCSMO:Pd10 composite,
\end{abstract}

R.C. Bhatt · S.K. Agarwal · V.P.S. Awana ( $\varangle)$

National Physical Laboratory (CSIR), New Delhi 110012, India

e-mail: awana@mail.nplindia.ernet.in

url: http://www.freewebs.com/vpsawana/

R.C. Bhatt - P.C. Srivastava

Department of Physics, Banaras Hindu University, Varanasi

221005, India which are far better than those reported for LCSMO:Ag10 composite.

Keywords Composite materials · Grain boundaries · Magnetoresistance $\cdot$ TCR $\cdot$ Magnetocaloric

\section{Introduction}

Colossal magneto-resistive $(C M R)$ manganites have been considered to be promising candidates for wide range of applications like magnetic sensors, bolometric devices and magnetic refrigeration [1-7]. The interesting physical properties of the manganites are known to originate from a complex interplay among structural, electrical, and magnetic degrees of freedom. Observation of the $C M R$ at low temperatures and under high magnetic fields $(\sim 7 \mathrm{~T})$ has restricted their practical usage so far. Efforts are being carried out to obtain sizable $M R$ values at low fields $(L F M R)$ and near room temperature $[8,9]$. Several manganite materials like LCBMO, LCSMO, PBMO and their composites with $\mathrm{Ag}_{2} \mathrm{O}$ and $\mathrm{PdO}$ have been investigated to explore their potential for magnetic sensing applications [8-13]. Besides, manganites have also shown good potential as active magnetic refrigerant $(A M R)$ near room temperature [10, 14-17].

Advantages of magnetic refrigeration include simple design, environmentally friendly components, and low operating costs [18]. When such materials are subjected to a varying magnetic field, there is an adiabatic change in the sample temperature or an isothermal change in the magnetic entropy exhibiting the magneto-caloric effect $(M C E)$. There are two key requirements for a material to possess good MCE. One is the large spontaneous magnetization and the other is the sharp drop in magnetization at Curie temperature. Magnetic 
Fig. 1 (a) Rietveld refined $X R D$ pattern of LCSMO and LCSMO:Pd10 samples. (b) Expanded view of (022) $X R D$ peak of LCSMO:Pd10 sample

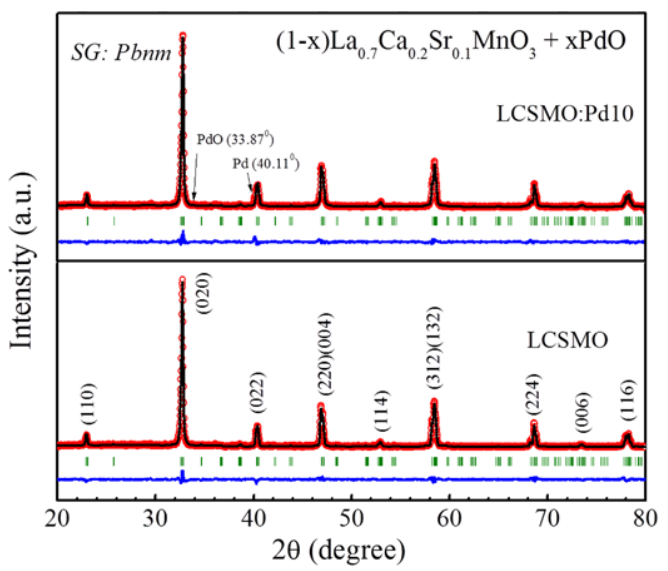

(a)

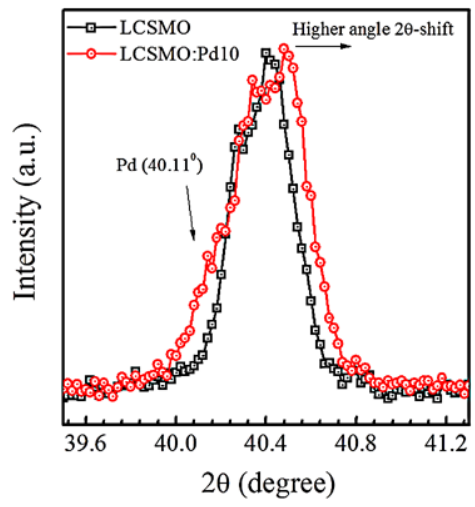

(b) refrigeration serves to be the best alternate to the conventional gas compression technique [19]. In magneto-caloric effect $(M C E)$ the cooling is achieved by demagnetizing the magnetized material and the cooling efficiency largely depends upon the magnetization value to the applied field. The magnetic entropy change $\left(\Delta S_{M}\right)$ is used to measure $M C E$ and it is required to be large over working temperature range. Gd and its alloys/compounds show high value of $\left|\Delta S_{M}\right|$ but are quite expensive $[18,20,21]$. The other intermetallic alloys/compounds exhibit high $\left|\Delta S_{M}\right|$ value but at high applied magnetic field, which restricts their practical applicability [22-25]. One problem is the required temperature range of operation. For household applications we need high value of $\left|\Delta S_{M}\right|$ near room temperature. Therefore we tried to overcome the said difficulties by using manganites as magnetocaloric material. Although comparable magnetocaloric effect has also been observed in charge ordered manganites, however, their lower charge ordering transition temperature and the high magnetic field requirement for the destruction of the antiferromagnetic $(A F M)$ phase and the following large $M C E$ restrict their usage at or close to room temperature. Therefore, manganite with magnetic transition close to room temperature is desired with good magnetocaloric and magneto-resistive properties at lower magnetic field application. In manganites, insulator to metal transition $\left(T_{p}\right)$ coexists with ferromagnetic to paramagnetic transition. The change in magnetic entropy increases with the sharpness of the magnetic transition and magnetization value. The sharpness of the transition can be increased by making grain boundaries conducting, which was initially nonmagnetic and insulating [12]. Thus we can expect to obtain enhanced properties in the composite material. Various manganite composites have been investigated for their efficacy towards magnetic sensing and refrigeration applications [8-17]. More recently, $\mathrm{La}_{0.7}\left(\mathrm{Ca}_{0.2} \mathrm{Sr}_{0.1}\right) \mathrm{MnO}_{3}: \mathrm{Ag}_{2} \mathrm{O}$ composite has been reported for its enhanced $M R$ and $M C E$ properties vis-à-vis the pristine material [10]. In the present communication we have explored $\mathrm{La}_{0.7}\left(\mathrm{Ca}_{0.2} \mathrm{Sr}_{0.1}\right) \mathrm{MnO}_{3}$ : $\mathrm{Pd}$ composite for its magnetic properties near room temperature and the results are compared with those on LCSMO:Ag composite.

\section{Experimental}

Polycrystalline $\mathrm{La}_{0.7}\left(\mathrm{Ca}_{0.2} \mathrm{Sr}_{0.1}\right) \mathrm{MnO}_{3} \quad$ (LCSMO) and $\mathrm{La}_{0.7}\left(\mathrm{Ca}_{0.2} \mathrm{Sr}_{0.1}\right) \mathrm{MnO}_{3}$ composite with $10 \% \mathrm{PdO}$ (LCSMO:Pd10) samples have been synthesized in air by the standard solid-state reaction route with starting stoichiometric mixture of $\mathrm{La}_{2} \mathrm{O}_{3}, \mathrm{CaCO}_{3}, \mathrm{SrCO}_{3}$ and $\mathrm{MnO}_{2}$ (all with $5 \mathrm{~N}$ purity). These ingredients were ground thoroughly, calcined at several steps of $900{ }^{\circ} \mathrm{C}$ for $18 \mathrm{~h}, 1100^{\circ} \mathrm{C}$ for $18 \mathrm{~h}, 1300{ }^{\circ} \mathrm{C}$ for $18 \mathrm{~h}$, respectively, and finally sintered at $1400^{\circ} \mathrm{C}$. Thus prepared sample was powdered and $\mathrm{PdO}$ was added and pelletized. Both the pristine and $\mathrm{PdO}$ composite samples were finally sintered at $1400^{\circ} \mathrm{C}$ for $30 \mathrm{~h}$ in furnace, cooling to room temperature. Phase purity and the lattice parameter refining of the samples were checked through $\mathrm{X}$-ray powder diffractometer using $\mathrm{CuK} \alpha$ radiation (Rigaku) and the Rietveld refinement program (Fullprof version). Scanning electron microscope (Ziess EVO MA-10) with attached energy dispersive spectroscopy $(E D S)$ was used for the surface morphology of the various samples. Resistivity and magnetization measurements of the samples were carried out in magnetic field up to $3 \mathrm{~T}$ using a Physical Properties Measurement System (PPMS-14T, Quantum Design).

\section{Results and Discussion}

Synthesized samples of the pristine $\mathrm{La}_{0.7}\left(\mathrm{Ca}_{0.2} \mathrm{Sr}_{0.1}\right) \mathrm{MnO}_{3}$ and $\mathrm{La}_{0.7}\left(\mathrm{Ca}_{0.2} \mathrm{Sr}_{0.1}\right) \mathrm{MnO}_{3}: \mathrm{PdO}$ composites have been analyzed for the phase formation by Rietveld refinement of their X-ray diffractograms. Figure 1(a) depicts the Rietveld 

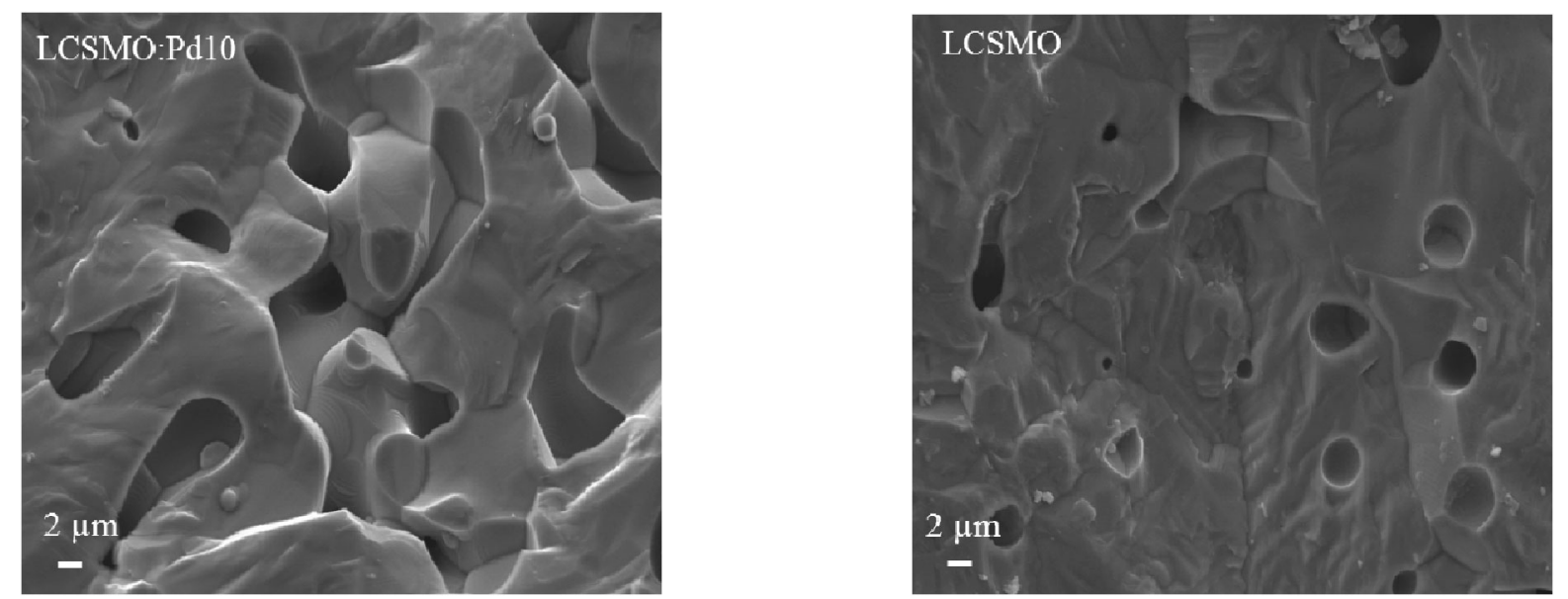

\section{(a)}
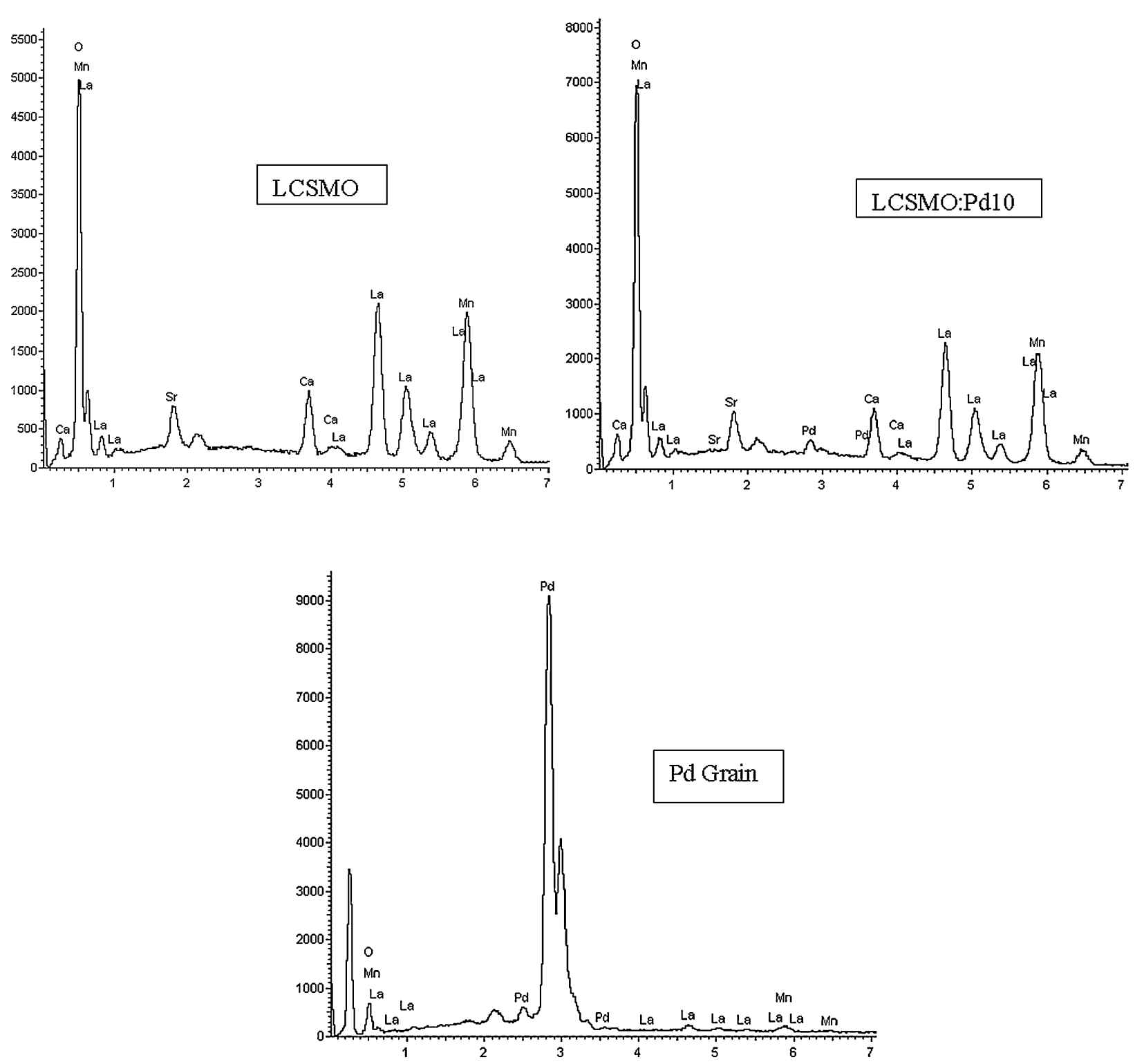

(b)

Fig. 2 (a) Scanning electron micrographs of LCSMO and LCSMO:Pd10 samples. (b) EDS patterns of LCSMO, LCSMO:Pd10 and Pd grain of LCSMO:Pd10 sample 
refined XRD patterns of LCSMO and LCSMO:Pd10 samples, confirming the presence of all the identified characteristic peaks and fitted in the orthorhombic Pbnm space group. The most intense peak of $\mathrm{PdO}$ at $33.87^{\circ}$ (JCPDS 41-1107) has been found absent in the XRD pattern of LCSMO:Pd10 sample due to the dissociation of PdO into metallic Pd. Rietveld refinement revealed practically no significant change in the lattice parameters and lattice volume with $\mathrm{PdO}$ addition (Table 1). However, Fig. 1(b) showing the $X R D$ peak of Pd at $40.11^{\circ}$ (JCPDS 46-1043) in LCSMO:Pd10 sample seems to merge with LCSMO main peak and the XRD pattern slightly shifts towards the higher angle, resulting in insignificant change in the lattice parameters of the composites (Table 1). Pd is not occupying any of the lattice sites because of its ionic size and coordination number $(C N)$ incompatibility with those of $\mathrm{Ca}^{+2}$ and $\mathrm{Sr}^{+2}$. Coordination number $(C N)$ of $\mathrm{Ca}^{+2}$ and $\mathrm{Sr}^{+2}$ in LCSMO is 9 whereas the available $\mathrm{CN}$ of $\mathrm{Pd}^{+2}$ is 4 and 6 .

Scanning electron micrographs (SEM) of pristine LCSMO and LCSMO:Pd10 composite samples are shown in Fig. 2(a). It is clear from the micrographs that no secondary phases have formed and that at such high sintering temperature the polycrystalline nature comprised of grain and grain boundaries almost disappears. These samples were not in a melted state and the observed behavior could be attributed to the coalescence of smaller grains and forming bigger size grains. Further, due to the higher sintering temperature of $1400{ }^{\circ} \mathrm{C}$ used in synthesis of the materials, $\mathrm{PdO}$ gets converted into metallic $\mathrm{Pd}$ (PdO dissociation temperature being $\left.\sim 750^{\circ} \mathrm{C}\right)[8,9,12,13]$. Elemental analysis performed using EDS attachment to SEM confirms the presence of Pd in the LCSMO:Pd10 composite (Table 2). Slight change observed in the oxygen stoichiometry of the composite material seemingly appears due to the oxygen re-

Table 1 Rietveld refined lattice parameters and volume of $\mathrm{La}_{0.7}\left(\mathrm{Ca}_{0.2} \mathrm{Sr}_{0.1}\right) \mathrm{MnO}_{3}: \mathrm{Pd}$

\begin{tabular}{lllll}
\hline Sample & $a(\AA)$ & $b(\AA)$ & $c(\AA)$ & Vol. $\left(\AA^{3}\right)$ \\
\hline LCSMO & $5.461(3)$ & $5.493(1)$ & $7.713(8)$ & 231.417 \\
LCSMO:Pd10 & $5.458(9)$ & $5.491(8)$ & $7.711(8)$ & 231.195 \\
\hline
\end{tabular}

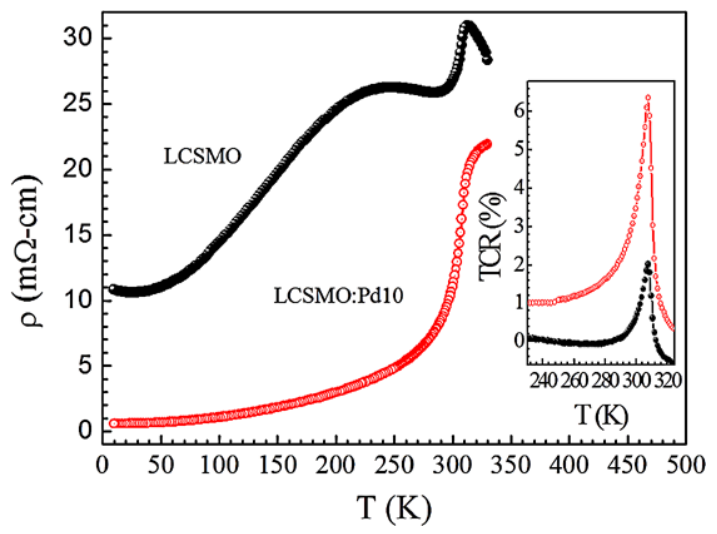

Fig. 3 Temperature-dependent resistivity for LCSMO and LCSMO: Pd10 samples. The inset shows the corresponding TCR of the samples

leased from the dissociation of $\mathrm{PdO}$ during the sintering process. EDS analysis of the shining region in LCSMO:Pd10 clearly shows the presence of Pd in the composite material (Fig. 2(b)). In LCSMO:Pd10 micrograph, the grain boundaries appear shining due to coating of the metallic Pd, which manifests the overall resistivity decrease of the composite (Fig. 3).

Temperature dependence of resistivity $\rho(T)$ of the samples is shown in Fig. 3 with their corresponding TCR in the inset. The hump around $230 \mathrm{~K}$ in the pristine LCSMO sample represents the conduction through grain boundaries which offer some resistive path to the carriers at lower temperatures. Incorporation of Pd makes grain boundaries conducting and therefore the overall resistivity of the LCSMO:Pd10 sample decreases [8, 9, 12, 13]. The hump disappears (due to this lower resistance) due to metallic $\mathrm{Pd}$ within grain boundaries and tunneling of the carriers across them. This grain boundary effect also increases the sharpness of the insulator-metal transition and in consequence the maximum temperature coefficient of resistance $T C R_{\mathrm{MAX}}$ also increases from $2 \%$ (at $305 \mathrm{~K}$ ) to $6.4 \%$ (at $308 \mathrm{~K}$ ) for LCSMO and LCSMO:Pd10, respectively.

The field-dependent magneto-resistance $(M R)$ of both the samples LCSMO and LCSMO:Pd10 is shown in Fig. 4. MR has been calculated using the formula $M R(\%)=\left[R_{0}-\right.$ $\left.R_{H} / R_{0}\right] \times 100$, where $R_{H}$ and $R_{0}$ are the resistances with
Table 2 Elemental analysis using $E D S$ for $\mathrm{La}_{0.7}\left(\mathrm{Ca}_{0.2} \mathrm{Sr}_{0.1}\right) \mathrm{MnO}_{3}: \mathrm{Pd}$ system

\begin{tabular}{lcc}
\hline Element & $\begin{array}{l}\mathrm{La}_{0.7}\left(\mathrm{Ca}_{0.2} \mathrm{Sr}_{0.1}\right) \mathrm{MnO}_{3} \\
(\text { Atomic \% })\end{array}$ & $\begin{array}{l}\mathrm{La}_{0.7}\left(\mathrm{Ca}_{0.2} \mathrm{Sr}_{0.1}\right) \mathrm{MnO}_{3}: \mathrm{Pd}_{10} \\
(\text { Atomic \% })\end{array}$ \\
\hline $\mathrm{O}$ & 59.26 & 64.7 \\
$\mathrm{Ca}$ & 4.01 & 3.52 \\
$\mathrm{Mn}$ & 21.48 & 18.2 \\
$\mathrm{Sr}$ & 1.87 & 1.85 \\
$\mathrm{La}$ & 13.39 & 10.94 \\
$\mathrm{Pd}$ & 0.00 & 0.78 \\
\hline
\end{tabular}




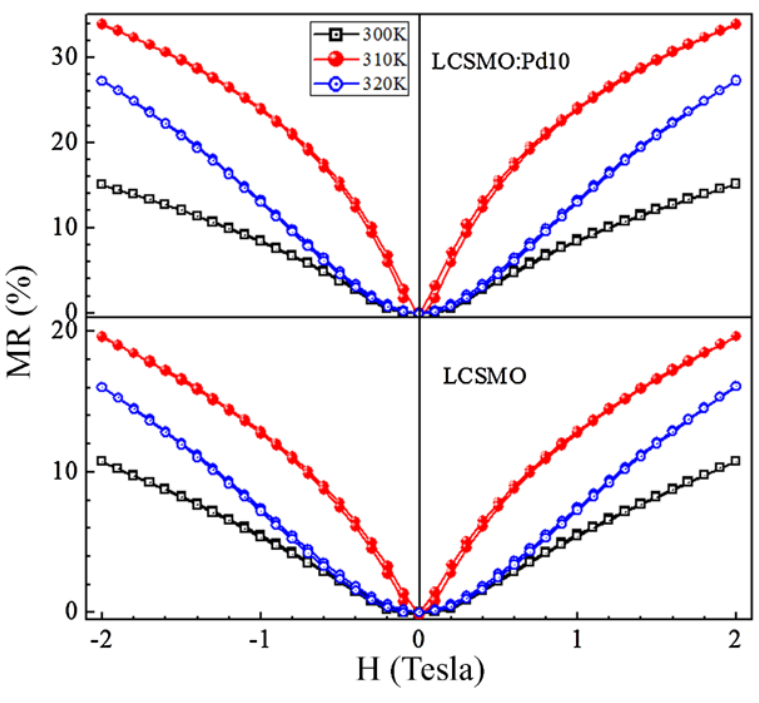

Fig. 4 Field-dependent $M R$ at 300, 310 and $320 \mathrm{~K}$ for LCSMO and LCSMO:Pd10 samples

and without the applied magnetic field. Figure 4 depicts the $M R$ values of the samples in the paramagnetic $(320 \mathrm{~K})$ and ferromagnetic $(300 \mathrm{~K})$ states, respectively. The maximum $M R$ of $12.9 \%(1 \mathrm{~T})$ and $19.6 \%(2 \mathrm{~T})$ has been observed in the pristine LCSMO close to its $T_{c}(310 \mathrm{~K})$. These values get enhanced to $24.1 \%(1 \mathrm{~T})$ and $33.9 \%(2 \mathrm{~T})$ with $\mathrm{PdO}$ addition. The observed augmentation in $M R$ can be ascribed to the conducting channels due to the metallic grain boundaries in the $\mathrm{PdO}$ composite. The applied magnetic field aligns the spins and thus surges the hopping of electron from $\mathrm{Mn}^{+3}$ to $\mathrm{Mn}^{+4}$ in the double exchange phenomena and consequently resistivity decreases and higher $M R$ gets generated.

The impact of $\mathrm{PdO}$ addition can also be noticed in the magnetization curve (Fig. 5) where temperature-dependent magnetization $[M(T)]$ and the sharpness of the Curie point are depicted in the inset for both LCSMO and LCSMO:Pd10 samples at a field value of 500 Oe. It can be seen that both the samples are excellent ferromagnets. With the addition of $\mathrm{PdO}$, magnetization value $(\mathrm{emu} / \mathrm{g})$ increases which increases the sharpness of the transition. Curie temperature $\left(T_{c}\right)$, however, remains unchanged at $\sim 309 \mathrm{~K}$, which has been ascribed to the unchanged stoichiometry of the LCSMO:Pd10 composite as confirmed by the X-ray Rietveld analysis.

The $\left|\Delta S_{M}\right|$ values at different temperatures have been calculated using the isothermal magnetization taken in the temperature range of 298 to $316 \mathrm{~K}$ at the regular interval of $2 \mathrm{~K}$ around $T_{c}$ as depicted in Fig. 6(a). The corresponding Arrott plots are shown in Fig. 6(b). The positive slopes for all the isotherms in Arrott plots show second-order magnetic transition. The temperature dependence of the magnetic entropy change is shown in Fig. 7. The maximum values of $\left|\Delta S_{M}\right|$ are found to be 4.0, 7.4 and $10.2 \mathrm{~J} \mathrm{~kg}^{-1} \mathrm{~K}^{-1}$ for LC-

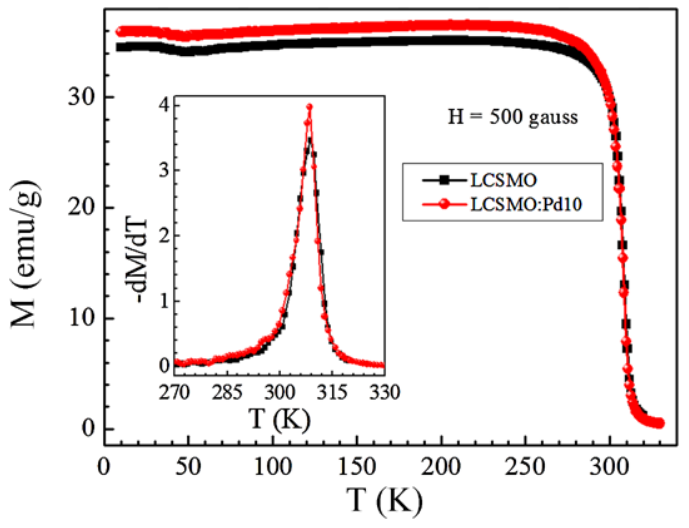

Fig. 5 Temperature-dependent $D C$ magnetization for LCSMO and LCSMO:Pd10 samples. The inset shows the corresponding $-d M / d T$

SMO, and 4.4, 8.2 and $11.7 \mathrm{~J} \mathrm{~kg}^{-1} \mathrm{~K}^{-1}$ for LCSMO:Pd10 at field values of 1,2 and $3 \mathrm{~T}$, respectively.

The magnetic entropy change $\left|\Delta S_{M}\right|$ values of both the samples in the present study have been compared with those reported earlier on LCSMO:Ag10 composite [10]. The $\left|\Delta S_{M}\right|$ values reported for LCSMO:Ag10 composite [10] were found to be 2.2, 3.8, 5.2 and $7.6 \mathrm{~J} \mathrm{~kg}^{-1} \mathrm{~K}^{-1}$ at $1,2,3$ and $5 \mathrm{~T}$ field values, respectively. The magnetic entropy values $\left|\Delta S_{M}\right|$ achieved for small magnetic field change $(0-3 \mathrm{~T})$ in the present samples (LCSMO and LCSMO:Pd10) are significantly higher than those reported in LCSMO, LCSMO single crystal and LCSMO:Ag10 composite $[5,10,15]$. These low field entropy values are also much higher when compared to manganites reported in the literature $[5,14,16]$. LCSMO:Pd composite material possessing a sizable $M R$ and high $\left|\Delta S_{M}\right|$ values therefore shows a promise of potential magnetic sensor and magnetic refrigerant close to room temperature.

\section{Conclusions}

Magneto-resistive and magneto-caloric properties of polycrystalline $\mathrm{La}_{0.7}\left(\mathrm{Ca}_{0.2} \mathrm{Sr}_{0.1}\right) \mathrm{MnO}_{3}$ and $\mathrm{La}_{0.7}\left(\mathrm{Ca}_{0.2} \mathrm{Sr}_{0.1}\right)$ $\mathrm{MnO}_{3}: 10 \% \mathrm{PdO}$ composites have been studied. Rietveld refinement of X-ray diffraction (XRD) patterns confirms their single-phase crystalline structure with orthorhombic Pbnm space group. Both the insulator-metal transition $\left(T^{\mathrm{IM}}\right)$ and Curie temperature $\left(T_{c}\right)$ are observed close to room temperature. While $T_{c}$ remained nearly unchanged, the peak value of the temperature coefficient of resistance (TCR $\mathrm{MAX})$ increased from 2 to $6.4 \%$ in the composites. Considerable improvement in both the magnetic $(M R)$ and magneto-caloric (MCE) properties of the pristine $\mathrm{La}_{0.7}\left(\mathrm{Ca}_{0.2} \mathrm{Sr}_{0.1}\right) \mathrm{MnO}_{3}$ (LCSMO) has been observed with PdO addition. The maximum MR of $12.9 \%(1 \mathrm{~T})$ and $19.6 \%(2 \mathrm{~T})$ has been observed close to its $T_{c}(309 \mathrm{~K})$ in the pristine LCSMO which 


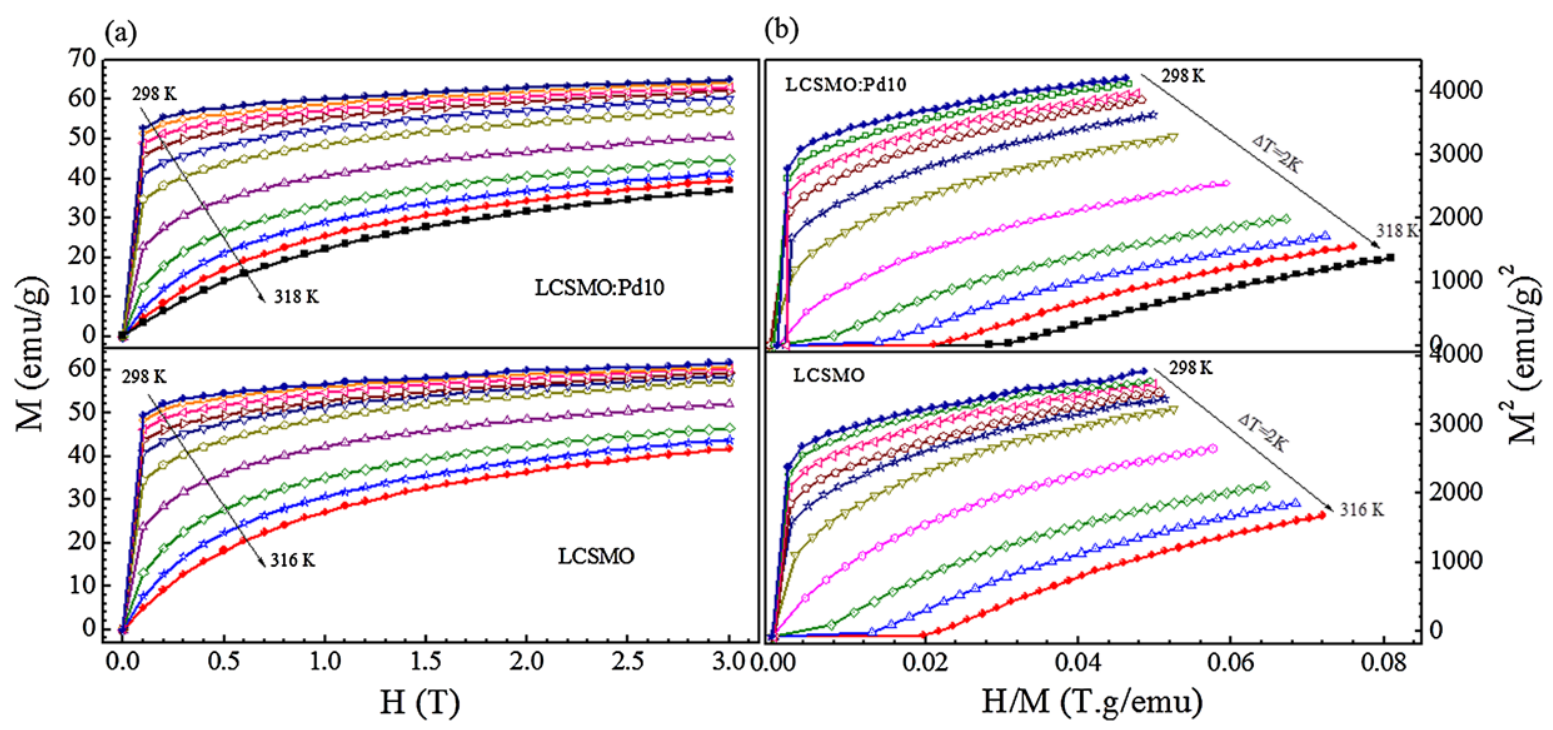

Fig. 6 (a) Isothermal magnetization $M(H)$ for LCSMO and LCSMO:Pd10 samples and (b) corresponding Arrott plots

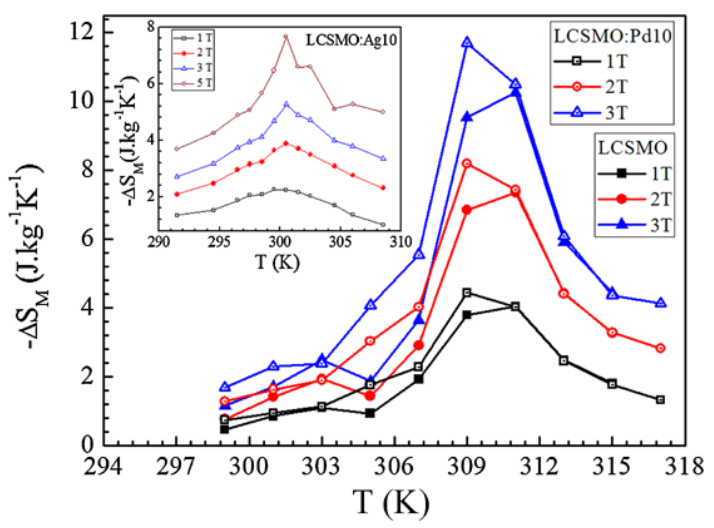

Fig. 7 Temperature dependence of magnetic entropy change $\left(\Delta S_{M}\right)$ for the pristine LCSMO and LCSMO:Pd10 composite at $H=1,2$ and $3 \mathrm{~T}$ fields. The inset depicts the $\Delta S_{M}$ data for LCSMO:Ag10 composite taken from Ref. [10] for comparison

got enhanced to $24.1 \%(1 \mathrm{~T})$ and $33.9 \%(2 \mathrm{~T})$ with the addition of $\mathrm{PdO}$.

The maximum values of magnetic entropy change $\left|\Delta S_{M}\right|$ of $11.7 \mathrm{~J} \mathrm{~kg}^{-1} \mathrm{~K}^{-1}(H=3 \mathrm{~T}), 8.2 \mathrm{~J} \mathrm{~kg}^{-1} \mathrm{~K}^{-1}(H=2 \mathrm{~T})$ and $4.4 \mathrm{~J} \mathrm{~kg}^{-1} \mathrm{~K}^{-1}(H=1 \mathrm{~T})$ for LCSMO:Pd10 are found to be much higher than in the LCSMO single crystal and the corresponding LCSMO:Ag composite reported earlier $[10,15]$. LCSMO:Pd composite material possessing a sizable $M R$ and high magnetic entropy value therefore shows an excellent possibility of a potential magnetic sensor and magnetic refrigerant close to room temperature.

Acknowledgements The authors thank the Director, National Physical Laboratory, New Delhi, for his support and encouragement. The authors R.C.B. and S.K.A. would like to thank the Council of Scientific and Industrial Research (CSIR), New Delhi, for the financial assistance under the Emeritus Scientist Scheme (Dr. S.K. Agarwal).

\section{References}

1. Jin, S., Tiefel, T.H., McCormack, M., Fastnacht, R.A., Ramesh, R., Chen, L.H.: Science 264, 413 (1994)

2. Haghiri-Gosnet, A.-M., Renard, J.-P.: J. Phys. D 36, R127 (2003)

3. Lacaze, A.F., Beranger, R., Bon Mardion, G., Claudet, G., Lacaze, A.A.: Cryogenics 23, 427 (1983)

4. Awana, V.P.S., Tripathi, R., Kumar, N., Kishan, H., Bhalla, G.L., Zeng, R., Sharath Chandra, L.S., Ganesan, V., Habermeier, H.U.: J. Appl. Phys. 107, 09D723 (2010)

5. Lin, G.C., Yu, X.L., Wei, Q., Zhang, J.X.: Mater. Lett. 59, 2149 (2005)

6. Huang, Y.-H., Yan, C.-H., Luo, F., Song, W., Wang, Z.-M., Liao, C.-S.: Appl. Phys. Lett. 81, 76 (2002)

7. Xu, Y., Memmert, U., Hermann, U.: Sens. Actuators A, Phys. 91, $26(2001)$

8. Yuan, X.B., Liu, Y.H., Wang, C.J., Mei, L.M.: Appl. Phys. Lett. 88, 042508 (2006)

9. Yuan, X.B., Liu, Y.H., Yin, N., Wang, C.J., Mei, L.M.: J. Phys. D 39, 1028 (2006)

10. Jha, R., Singh, S.K., Kumar, A., Awana, V.P.S.: J. Magn. Magn. Mater. 324, 2849 (2012)

11. Panwar, N., Pandya, D.K., Agarwal, S.K.: J. Phys. Condens. Matter 19, 456224 (2007)

12. Panwar, N., Pandya, D.K., Agarwal, S.K.: J. Phys. D, Appl. Phys. 40, 7548 (2007)

13. Xiong, C., Hu, H., Xiong, Y., Zhang, Z., Pi, H., Wu, X., Li, L., Wei, F., Zheng, C.: J. Alloys Compd. 479, 357 (2009)

14. Phan, M.H., Yu, S.C.: J. Magn. Magn. Mater. 308, 325 (2007)

15. Phan, M.H., Yu, S.C., Hur, N.H.: Appl. Phys. Lett. 86, 72504 (2005)

16. Phan, M.H., Tian, S.B., Hoang, D.Q., Yu, S.C., Nguyen, C., Ulyanov, A.N.: J. Magn. Magn. Mater. 258, 309 (2003)

17. Bhatt, R.C., Singh, S.K., Srivastava, P.C., Agarwal, S.K., Awana, V.P.S.: J. Alloys Compd. 580, 377 (2013)

18. Gschneidner, K.A., Pecharsky, V.K.: Annu. Rev. Mater. Sci. 30, 387 (2000)

19. McMichael, R.D., Ritter, J.J., Shull, R.D.: J. Appl. Phys. 73, 6946 (1993)

20. Yu, B.F., Gao, Q., Zhang, B., Meng, X.Z., Chen, Z.: Int. J. Refrig. 26, 622 (2003) 
21. Brown, G.V.: J. Appl. Phys. 47, 3673 (1976)

22. Hu, F.X., Shen, B.G., Sun, J.R., Wu, G.H.: Phys. Rev. B 64, 132412 (2001)

23. Wada, H., Tanabe, Y.: Appl. Phys. Lett. 79, 3302 (2001)
24. Fujieda, S., Fujita, A., Fukamichi, K.: Appl. Phys. Lett. 81, 1276 (2002)

25. Tegus, Q., Bruck, E., Buschow, K.H., de Boer, F.R.: Nature 415, $150(2002)$ 